\title{
Corona-Pandemie: Entwicklungsprobleme bei Schulneulingen nachweisbar
}

Als Ärztin für Kinder- und Jugendmedizin leite ich im Gesundheitsamt der Landeshauptstadt Düsseldorf das Sachgebiet Kinder- und Jugendgesundheit. Unser Team von Kinderkrankenschwestern und Kinder- und Jugendärztinnen untersucht die Düsseldorfer Kinder zur Einschulung, aber auch wegen anderer Fragestellungen, besonders schulisch und wegen Eingliederungshilfe. Neben der optimalen Beratung und Entscheidung in jedem Einzelfall tragen wir zur Gesundheitsberichterstattung („Daten für Taten“) bei und wollen so Kindergesundheit und Kinderschutz fördern natürlich auch während der COVID-19-Pandemie. Besonders wichtig sind uns aus sozialen (und medizinischen) Gründen benachteiligte Kinder und Jugendliche, die sozialkompensatorisch unterstützt werden.

\section{Corona-Pandemie: Sorgen um die Entwicklung von Schulneulingen}

Das Thema der pandemiebedingten Entwicklungsprobleme bei Kindern wird sowohl von niedergelassenen und von im öffentlichen Gesundheitsdienst tätigen Kinder- und Jugendärztinnen und -ärzten, als auch von vielen anderen Akteuren aus den Bereichen Gesundheit, Jugendhilfe und Bildungswesen seit längerem höchst besorgt angesprochen. In den unterschiedlichsten Kontexten wird deutlich, dass viele Kinder unter den Bedingungen von Corona und Lockdown, der Einschränkung von institutionalisierter Bildung und Betreuung und unter erheblicher zusätzlicher Belastung ihrer Eltern Entwicklungsschwierigkeiten entwickelt haben.

In der Reihenuntersuchung der Schulneulinge und unter Einbeziehung der Ergebnisse des SOPESS (siehe unten) gelang es frühzeitig, einen negativen Effekt für bestimmte Kinder in Düsseldorf tatsächlich nachzuweisen. 


\section{Tätigkeiten des Sachgebietes Kinder- und Jugendgesundheit}

Im Sachgebiet Kinder- und Jugendgesundheit der Landeshauptstadt Düsseldorf wird normalerweise der Jahrgang der Düsseldorfer einzuschulenden Kinder zur Schulneulingsuntersuchung eingeladen. Zur Untersuchung gehören die individuelle Beurteilung der Gesundheit des Kindes in verschiedensten Aspekten sowie die Empfehlungen der untersuchenden (Fach-)ärztin; diese stützt sich unter anderem auch, wie überall in NRW und mittlerweile in vielen anderen Bundesländern, auf das SOPESS (sozialpädiatrisches Entwicklungsscreening für Schuleingangsuntersuchungen). SOPESS umfasst standardisierte, in Punkten bewertete Aufgaben und damit die Grundlage für eine Einteilung des jeweiligen Entwicklungsbereiches als unauffällig, grenzwertig oder auffällig. Erfasst werden die Bereiche Selektive Aufmerksamkeit, Zählen, Simultanerfassung und Mengenvergleich, Visuomotorik, Visuelle Wahrnehmung, Präpositionen, Pluralbildung, Pseudowörter, Artikulation und Körperkoordination, siehe auch Tab. 1. Es handelt sich um ein reliables, gut validiertes und in NRW flächendeckend genutztes und auch sonst weit verbreitetes Screeningprogramm (Daseking et al. 2009) ${ }^{1}$.

Zusätzlich werden im Sachgebiet Kinder- und Jugendgesundheit Untersuchungen zahlreicher anderer Kinder wegen schulischer Fragestellungen und im Rahmen der Eingliederungshilfe für Behinderte und von Behinderung bedrohte Kinder sowie in Kindergärten durchgeführt. Damit kommen die Kinder- und Jugendärztinnen und Gesundheits- und Kinderkrankenpflegerinnen des Sachgebietes mit einem sehr breiten Spektrum von Kindern, deren Familien und Lebenswelten aus ganz Düsseldorf in Kontakt.

\section{Corona-Situation im Sachgebiet Kinder- und Jugendgesundheit}

Die regulär im Herbst 2019 begonnene Untersuchung der August 2020 einzuschulenden Kinder musste aufgrund des Lockdowns am 13. März 2020 zunächst beendet werden. Bis dahin waren 2.969 Kinder untersucht worden. Unter sorgfältigen

\footnotetext{
1 Detaillierte Angaben hierzu finden sich auch beim Landeszentrum Gesundheit NRW, das auch dieses Programm mitentwickelte und die nötigen Unterlagen dazu den Kommunen zur Verfügung stellt (https://www.lzg.nrw.de/nocms/jahresberichte/reports/2012/up3/sopess_punkte/ sopess.html. Zugriff am 8. Oktober 2021).
} 
und situationsangepassten Hygienemaßnahmen konnten neben sehr dringenden anderen Untersuchungen später nur noch 80 Schulneulinge untersucht werden.

Im Herbst 2020 begann ebenfalls unter oben genannten Bedingungen die Untersuchung von im Jahr 2021 einzuschulenden Kindern. Die zu beachtenden umfassenden Hygienemaßnahmen und die sonstigen Tätigkeiten der Mitarbeiterinnen reduzieren die Anzahl der zur Verfügung stehenden Termine so, dass nur erheblich weniger Kinder eingeladen werden können. Im Laufe der Zeit konnten diese Effekte teilweise aufgefangen werden, aber letztlich wird nur ein kleinerer Teil der Schulneulinge 2021 untersucht werden können.

\section{Erste Alarmzeichen}

Schon im September 2020, bei Beginn der Untersuchungen der 2021 einzuschulenden Kinder sahen die Mitarbeiterinnen bei der Anamneseerhebung, dass ein Kindergartenbesuch kaum noch stattgefunden hatte und manche Eltern ihre Kinder deswegen komplett abgemeldet hatten. Auch Spezialuntersuchungen wie Vorstellungen in sozialpädiatrischen Zentren hatten wegen Aufschiebens der Termine oft nicht stattgefunden, Therapien waren teils langfristig unterbrochen worden. Die medizinische Basisversorgung inklusive Vorsorgeuntersuchungen und Impfungen hingegen war, sicher mit gewissen zeitlichen Verzögerungen, nachweisbar weitergelaufen.

Die Untersucherinnen bemerkten dann auch rasch, dass in allen Bereichen, besonders aber bzgl. der sprachlichen Fähigkeiten bzw. der Beherrschung des Deutschen gegenüber den Vorjahren deutlich reduzierte Ergebnisse vorlagen.

\section{Untersuchungskollektive und Ergebnisse}

Unsere aktuelle vergleichende Zwischenauswertung der ersten 782 untersuchten, 2021 einzuschulenden Kinder zeigt, dass die allgemeine Besorgnis nur zu gerechtfertigt ist.

Wie seit Jahren üblich, wurden auch 2020 direkt in der Anfangsphase der Schulneulingsuntersuchungen (jetzt bzgl. der Einschulung 2021) aus sozialkompensatorischen Gründen ganz überproportional Kinder mit gesundheitlichen und sozialen Gefährdungen und damit Kinder, bei denen Besonderheiten wegen medizinischer oder sozialer Faktoren zu erwarten sind, untersucht. Die beiden Gruppen der jeweils ersten 782 Kinder, die zur Einschulung 2020 im Herbst 2019 noch vor Beginn der Corona-Pandemie untersucht wurden (29. August bis zum 
Tab. 1: SOPESS-Kriterien

\begin{tabular}{lllllll}
\hline $\begin{array}{l}\text { Punktwert je SOPESS- } \\
\text { Parameter }\end{array}$ & $\begin{array}{l}\text { auf- } \\
\text { fällig }\end{array}$ & $\begin{array}{l}\text { grenz- } \\
\text { wertig }\end{array}$ & $\begin{array}{l}\text { unauf- } \\
\text { fällig }\end{array}$ & $\begin{array}{l}\text { maximal } \\
\text { erreich- } \\
\text { bar }\end{array}$ & $\begin{array}{l}\overline{\boldsymbol{X}} \text { Schul- } \\
\text { neulinge } \\
\mathbf{2 0 2 0}\end{array}$ & $\begin{array}{l}\overline{\boldsymbol{X}} \text { Schul- } \\
\text { neulinge } \\
\mathbf{2 0 2 1}\end{array}$ \\
\hline $\begin{array}{l}\text { Selektive Aufmerksamkeit } \\
\text { Zählen }\end{array}$ & $0-10$ & $11-13$ & $14-29$ & 29 & 15,3 & 13,71 \\
Simultanerfassung und & $0-12$ & $13-16$ & $17-20$ & 20 & 15,94 & 13,82 \\
Mengenvergleich & $0-10$ & $11-13$ & $14-16$ & 16 & 12,73 & 11,22 \\
Visuomotorik & & & & & & \\
Visuelle Wahrnehmung & $0-4$ & $5-6$ & $7-12$ & 12 & 6,57 & 5,87 \\
$\begin{array}{l}\text { Präpositionen } \\
\text { Pluralbildung }\end{array}$ & $0-8$ & $9-10$ & $11-15$ & 15 & 11,02 & 9,86 \\
$\begin{array}{l}\text { Pseudowörter } \\
\text { Körperkoordination }\end{array}$ & $0-4$ & 5 & $6-8$ & 8 & 4,9 & 3,63 \\
Deutsch & $0-3$ & $4-5$ & $6-7$ & 7 & 4,16 & 3,15 \\
\hline
\end{tabular}

SOPESS-Beurteilungskriterien sowie durchschnittliche absolute Punktzahlen der jeweils ersten 782 untersuchten Schulneulinge 2020 und 2021 (2 Spalten ganz rechts). Zum Parameter „Deutsch“ siehe Text.

25. November 2019) und die zur Einschulung 2021 ab Herbst 2020 untersucht wurden (7. September 2020 bis 17. März 2021) sind geeignete Vergleichsgruppen. Dies wird an der hohen, fast identischen Anzahl (422 versus 429) von Kindern aus Wohnbereichen mit überdurchschnittlichem (hohem oder sehr hohem) sozialem Handlungsbedarf (Golschinski 2017) offenbar².

Tabelle 1 zeigt die Punktwerte für alle SOPESS Parameter im Vergleich der beiden Gruppen. Es zeigt sich, dass die absoluten Punktewerte bei allen Parametern gesunken sind. So haben beispielsweise die ersten 782 untersuchten Schulneulinge 2020 im Durchschnitt 4,9 Punkte im Test Präpositionen erreicht, während die ersten 782 untersuchten Schulneulinge 2021 in diesem Test durchschnittlich nur 3,63 Punkte erreicht haben.

Für eine weitergehende Betrachtung wurden jeweils die Durchschnittspunktwerte der 782 ersten Schulneulinge 2020 als 100\%-Werte zu Grunde gelegt und die Durchschnittswerte der entsprechenden ersten Schulneulinge 2021 dazu in Beziehung gesetzt. Kinder, die im SOPESS entsprechende Aufgaben gar nicht lösen konnten bzw. nicht zur Mitarbeit bereit waren, erhielten 0 Punkte.

Bei der selektiven Aufmerksamkeit (maximale Punktzahl 29) sank der Durchschnittswert von 15,3 auf 13,71 Punkte und damit um 10,39 \%. Das Zählen (Maxi-

2 Zu den Kategorien der sozialräumlichen Gliederung, die vom Jugendamt der Landeshauptstadt Düsseldorf initiiert wurde, siehe auch Golschinski (2017). 
mum 20) sank mit den Durchschnittswerten von 15,94 auf 13,82 Punkte und damit um 13,3\%. Bzgl. Simultanerfassung und Mengenvergleich (erreichbares Maximum 16) reduzierte sich der Durchschnittswert von 12,73 auf 11,22 Punkte, also um 11,86\%. Im motorischen Bereich war in der Visuomotorik (maximal 12 Punkte) ein Absinken von durchschnittlich 6,57 auf 5,87 Punkte, also um 10,65\% zu verzeichnen. Im Bereich visuelles Wahrnehmen reduzierten sich die Punktwerte (Maximum 15) von 11,02 auf 9,86 Punkte und damit um 10,53\%.

Im Bereich Sprache reduzierten sich die Punktwerte für den Gebrauch von Präpositionen (maximal können 8 Punkte erzielt werden) von 4,90 auf 3,63 Punkte und damit um 25,92\%. Beim Bilden des Plurals (maximal 7 Punkte möglich) wurde eine Reduktion von 4,16 auf 3,15 Punkte und damit um 24,28 \% beobachtet. Das Nachsprechen von Pseudowörtern (möglich sind 6 Punkte) reduzierte sich von 4,48 auf 3,49 Punkte und damit um 12,05\%. Die Einschätzung der allgemeinen kommunikativen Fähigkeiten im Deutschen durch die Untersucherin auf einer Skala von 1 (keine Deutschkenntnisse) bis 5 (spricht flüssig und fehlerfrei Deutsch) reduzierte sich von 3,72 auf 3,25 Punkte und damit um 12,63\%.

In der Körperkoordinationsaufgabe (Seithüpfen, praktisch mögliches Maximum um 32) reduzierte sich der Durchschnittswert von 10,42 auf 8,69 Punkte und damit um 16,6\%. Abb. 1 illustriert die prozentuale Reduktion der Punktewerte für die Parameter des sozialpädiatrischen Screenings.

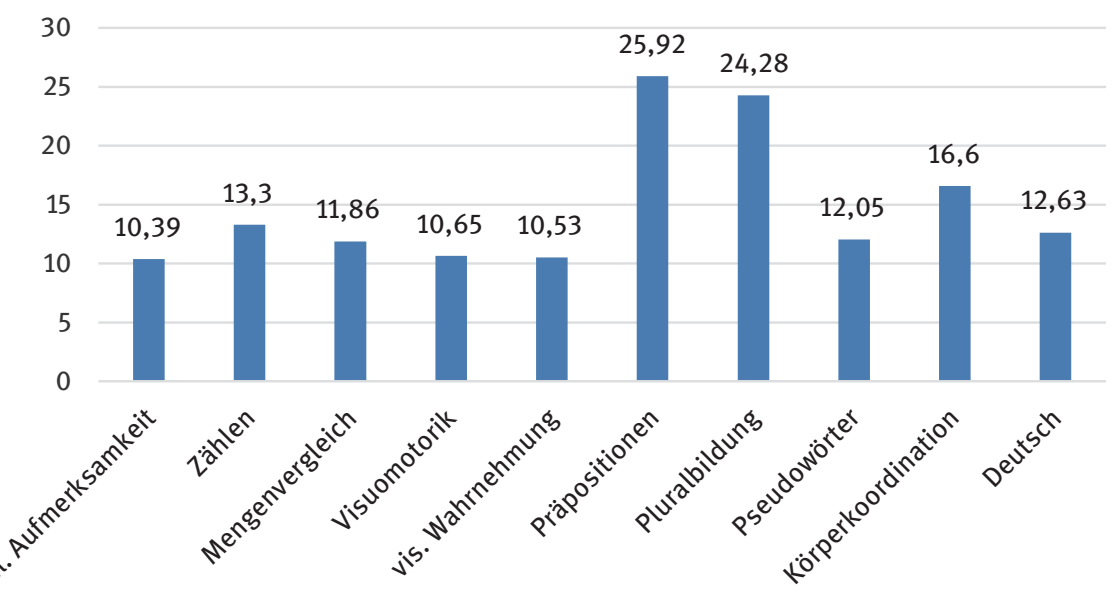

Abb. 1: Ausmaß des Verlustes an Punkten im SOPESS. Ergebnisse der jeweils ersten 782 untersuchten Schulneulinge 2021 gemessen an der Vergleichskohorte 2020, deren Werte als $100 \%$. Zum Parameter „Deutsch“ siehe Text. 
Der durchschnittliche Body-Mass-Index stieg außerdem von $16,16 \mathrm{~kg} / \mathrm{m}^{2}$ auf $16,57 \mathrm{~kg} / \mathrm{m}^{2}$ und somit um $2,5 \%$.

Allerdings fanden sich auch positive Entwicklungen. Die dokumentierte Inanspruchnahme der Vorsorgeuntersuchung U9 wurde bei den Schulneulingen 2020 bei 607 Kindern beobachtet und bei den Schulneulingen 2021 bei 628 Kindern. Damit fand sich sogar ein leichter Anstieg um 3,5\%. Die durchschnittlich dokumentierte Anzahl der Impfungen gegen Tetanus stieg ebenfalls leicht, von 3,92 auf 4 um ca. $2 \%$.

\section{Zusammenfassung und Ausblick}

Gegenüber den ersten 782 Kindern zur Einschulung 2020, die im Herbst 2019 noch vor Beginn der Corona-Pandemie untersucht wurden, zeigen sich bei den ersten 782 untersuchten Schulneulingen 2021 zwar erfreulich stabile Werte bezüglich der Teilnahme an Vorsorgeuntersuchungen und Impfungen und eine (noch!) geringe Zunahme des BMI. Andererseits wurden erhebliche Einbußen in den mit dem SOPESS dokumentierten Parametern des Entwicklungsstatus beobachtet. Erfasst wurden dabei ganz überproportional Kinder mit medizinischen und/oder mit sozialen Risiken. Zu Corona-Pandemie-Effekten bei weniger belasteten oder unbelasteten Kindern können wir keine Aussagen machen.

Zumindest in dieser speziellen Gruppe erweist sich also die allgemeine Besorgnis als gerechtfertigt. Eine langfristige Intensivierung aller wirksamen Fördermaßnahmen ist unbedingt erforderlich, besonders im Bereich der Sprache. Sowohl Sprachtherapien als auch Deutschförderung, auch im Setting der Kindergemeinschaftseinrichtungen, müssen ausgebaut werden. Wir alle dürfen jedoch auch dann von den Kindern kein „mal eben wieder aufholen“ einfordern, sondern langfristiges, gut überprüftes, geduldiges Handeln ist nötig. Die Reihenuntersuchungen der Kinder- und Jugendärztinnen und -ärzte des öffentlichen Gesundheitsdienstes können hier auch in Zukunft wertvolle Informationen beitragen. Ihre Untersuchung des (fast) kompletten Jahrgangs der Schulneulinge bietet die Basis für den querschnittsartigen, aber auch den longitudinalen und auch interkommunalen und Subgruppen berücksichtigenden Vergleich der Entwicklung durch die SOPESS-Parameter, aber auch weiterer gesundheitlicher Indikatoren (wie Inanspruchnahme der Vorsorgeuntersuchungen und des Impfangebotes, Körpermaße, Sehtest, Hörtest, chronische Erkrankungen, Behinderungen) und damit eine Handlungsgrundlage für gezielte Interventionsmaßnahmen. 
Kernaussagen: Zumindest in einem Kollektiv von Schulneulingskindern mit erhöhtem gesundheitlichem und/oder sozialem Risiko wurde die Entwicklung durch die Pandemie messbar gebremst. Besonders ausgeprägt ist dies im Bereich Sprache. Die Reihenuntersuchung der Schulneulinge mit dem SOPESS (sozialpädiatrisches Entwicklungsscreening für Schuleingangsuntersuchungen) ist geeignet, dies nachzuweisen.

Danksagung: Diese Ergebnisse konnten nur auf der Basis der kontinuierlichen und äußerst engagierten und sachkundigen Arbeit des gesamten Teams Kinderund Jugendgesundheit, Gesundheitsamt, Landeshauptstadt Düsseldorf entstehen. Danke dafür!

Frau Dr. phil. Simone Weyers vom Institut für Medizinische Soziologie danke ich für die Einladung zur Erstellung dieses Beitrages und die umfassende Hilfestellung.

\section{Literatur}

Daseking M, Petermann F, Roske D, Trost-Brinkhues G, Simon K, Oldenhage M (2009) Entwicklung und Normierung des Einschulungsscreenings SOPESS (Development and standardisation of the social-pediatric screening SOPESS). Gesundheitswesen 71:648-655. https:// doi.org/10.1055/s-0029-1239511.

Golschinski M (2017) Sozialräumliche Gliederung; Fortschreibung 2017. https://www. duesseldorf.de/fileadmin/Amt13/presseanhang/Sozialraeumliche-GliederungFortschreibung2017.pdf. Zugriff am 4. Oktober 2021. 
